\title{
Ausgefallene Sitzungen in der Praxispsychiatrie (Absentismus)
}

\author{
P. Hauser*
}

In einer Stichprobe von 342 psychiatrischen und kinder- und jugendpsychiatrischen Praxen wurde während zwei Erfassungsperioden die Anzahl und die Dauer der ausgefallenen Sitzungen untersucht (Absentismus).

Der Anteil von abgesagten und ausgefallenen Sitzungen betrug 12,8\%, der Anteil der unabgemeldet ferngebliebenen und der kurzfristig abgesagten Sitzungen betrug $8,5 \%$.

Diese Ergebnisse belegen, dass der Absentismus einen wesentlichen Faktor in der Praxispsychiatrie darstellt und daher die Produktivität wesentlich beeinträchtigt.

\footnotetext{
* Ständige Tarifkommission STK der Foederatio Medicorum Psychiatricorum et Psychotherapeuticorum FMPP
}

1 Brunner HH. Zur Infrastrukturund Arbeitszeiterhebung in der Psychiatrie. Schweiz Ärztezeitung 2002;83(25):1272.

2 Lachenmeier H. Infrastrukturund Arbeitszeiterhebung Psychiatrie. Schweiz Ärztezeitung 2002;83(25):1305-9.

Korrespondenz:

Dr. med. Philipp Hauser

Renggerstrasse 46

CH-5000 Aarau

E-Mail: philipp.hauser@hin.ch

\section{Einleitung}

Die Arbeit der praktizierenden Fachärzte für Psychiatrie und Psychotherapie sowie der Fachärzte für Kinder- und Jugendpsychiatrie und -psychotherapie ist bezüglich Klientel, Arbeitsabläufen und Infrastruktur besonderen Bedingungen unterworfen. Sie kann nur bedingt mit anderen Berufsgruppen der praktizierenden Ärzteschaft verglichen werden. Die Tatsache dieser besonderen Arbeitsbedingungen ist im Bereich der Ärzteschaft ebenso wie bei den Versicherern noch wenig bekannt. Dies zeigte sich in den bisherigen tarifpolitischen Verhandlungen, wo es sehr schwierig war, Akzeptanz für die Besonderheiten der Praxispsychiatrie zu erreichen.

Daher haben die beiden psychiatrischen Fachgesellschaften (SGP und SGKJPP) verschiedene Bereiche ihrer praktischen Arbeitstätigkeit mit neuen Erhebungen beleuchtet und im Verlaufe des Jahres 2001 gezielte Studien in Auftrag gegeben. Mittlerweile sind wichtige Resultate dieser Studien bereits publiziert worden $[1,2]$.

Die vorliegende Studie fokussiert auf einen speziellen Aspekt der psychiatrischen Arbeit:

Der Anteil abgesagter sowie unabgesagt ferngebliebener Konsultationen (künftig mit «Absentismus» bezeichnet) ist im Bereich der Psychiatrie erfahrungsgemäss sehr hoch. Dies ist auf die spezielle Pathologie der Klientel einer psychiatrischen Praxis zurückzuführen, in welcher häufig die Behandlungscompliance eine besondere Schwierigkeit darstellt und damit auch zu einem charakteristischen Teil der psychiatrischen Behandlung wird. Erschwert wird dieses Phänomen, da in der Psychiatrie die ausfallen- den Sitzungen erstens von wesentlich längerer Dauer sind und zweitens durch die Organisationsstruktur einer psychiatrischen Praxis nicht kurzfristig aus dem Wartezimmer kompensiert werden können.

Das Ausmass dieses Absentismus beeinflusst die Produktivität der Arbeit in der psychiatrischen Praxis, da ausfallende Sitzungen gegenüber den Versicherungsträgern nicht in Rechnung gestellt werden können. Damit wird diese ausfallende Zeit für den praktizierenden Psychiater zur nicht produktiven Zeit.

Die vorliegende Studie setzt sich zum Ziel, das Ausmass des Absentismus in der psychiatrischen Praxis zu erfassen. Die Erhebung soll eine hohe Anzahl von Praxen erfassen, um repräsentativ zu sein. Die Resultate der Studie sollen als Grundlage in die künftigen Verhandlungen für Tarifgestaltung in der Psychiatrie einfliessen. Von seiten der Versicherer wurde der Absentismus als die Produktivität beeinflussender Faktor in der Praxispsychiatrie anerkannt. Die technische Durchführung der Erhebung übernahm verdankenswerterweise der Tarifdienst FMH.

\section{Material und Methode}

Der FMH-Tarifdienst führte die Erhebung über den Absentismus in der Psychiatrie im Jahre 2001 für zwei Perioden von je zwei Wochen Dauer durch. Die erste Periode (Periode 1) dauerte vom 7. bis zum 19. Mai 2001, die zweite Periode (Periode 2) vom 2. bis zum 14. Juli 2001. Erhoben wurden die Anzahl und die Zeitdauer der Sitzungen. Die ausgefallenen Sitzungen wurden in drei verschiedenen Kategorien erfasst:

1. unabgemeldet ausgefallene Sitzungen;

2. kurzfristig (innerhalb von 48 Stunden) abgemeldete Sitzungen;

3. langfristig abgemeldete Sitzungen.

Die beiden Erfassungsperioden wurden so gelegt, dass Periode 1 in eine erfahrungsgemäss arbeitsintensive Zeit, die Periode 2 bewusst in die Ferienzeit gelegt wurde. Damit konnten verschiedene Bedingungen erfasst werden. 
Tabelle 1

Stichprobengrössen

\begin{tabular}{lcccc} 
& Periode $\mathbf{1}$ & $\%$ & Periode 2 & $\%$ \\
\hline Versandte Erhebungsbogen & 342 & 100 & 342 & 100 \\
\hline Rücklauf & 238 & 69,6 & 91 & 26,6 \\
\hline Plausibilitätstest nicht bestanden & 59 & 17,2 & 27 & 7,9 \\
\hline Ausgewertet & 179 & 52,4 & 64 & 18,7
\end{tabular}

3 FMH-Ärztestatistik 2001. Schweiz Ärztezeitung 2002; 83(18):871-90.
Die Studienteilnehmer protokollierten täglich die während der beschriebenen Perioden erfolgten Zahlen bezüglich gesamter Anzahl durchgeführter Sitzungen und der erwähnten Kategorien der ausgefallenen Sitzungen sowie deren Zeitdauer.

Es wurde nach Zufallsprinzip aus dem Verzeichnis sämtlicher praktizierender Mitglieder der beiden Fachgesellschaften SGP ( $=1433)$ [3] sowie SGKJPP ( $\mathrm{N}=287$ ) [3] eine Stichprobe von total 342 Einzelpraxen ausgewählt. Davon waren 224 aus der deutschen, 108 aus der französischen und 12 aus der italienischen Schweiz. Die angeschriebene Stichprobe entspricht 19,9\% der gesamten Zahl praktizierender Mitglieder.

Der Rücklauf der beiden Erfassungsperioden war sehr unterschiedlich. Aus Periode 1 wurden 238 (69,6\%) der verschickten Fragebogen zurück- gesandt. Aus Periode 2 sind hingegen nur noch 91 Fragebogen (26,6\%) eingegangen (Tab. 1).

Als Plausibilitätstest mussten die Anzahl und die Dauer der Sitzungen folgenden Kriterien genügen: Die Anzahl der durchgeführten Sitzungen musste im Erhebungszeitraum zwischen 30 und 90 Sitzungen liegen, musste grösser sein als die Summe der drei Kategorien ausgefallener Sitzungen und musste doppelt so gross sein wie die Summe der unabgemeldeten Sitzungen und der kurzfristig abgemeldeten Sitzungen. Die Gesamtdauer der ausgefallenen Sitzungen durfte 1000 Minuten nicht überschreiten.

\section{Resultate}

\section{Plausibilitätstest}

Durch die Plausibilisierung schieden in erster Linie Studienteilnehmer mit niedrigem Beschäftigungsgrad aus. Zusätzlich wurden 6 isolierte Ausreisser eliminiert, die fälschlicherweise unter der Rubrik «Dauer der ausgefallenen Sitzungen» die Gesamtdauer der stattgefundenen Sitzungen der Erhebungsperiode einsetzten.

Die durch diese Plausibilisierungen ausgeschiedenen Studienteilnehmer betrugen in Periode 1: 17,2\%, in Periode 2: 7,9\%. Damit betragen die ausgewerteten Anteile der Stichprobe in Periode 1: 52,4\%, in Periode 2: 18,7\%.

Tabelle 2

Ausgefallene Sitzungen.

\begin{tabular}{|c|c|c|c|c|c|c|c|}
\hline & $\begin{array}{l}\text { Anzahl } \\
\text { Praxen }\end{array}$ & $\begin{array}{l}\text { Anzahl } \\
\text { durchgeführte } \\
\text { Sitzungen }\end{array}$ & $\begin{array}{l}\text { Unabgemeldete } \\
\text { Absenzen }\end{array}$ & $\begin{array}{l}\text { Kurzfristig } \\
\text { abgesagt } \\
(<48 \mathrm{~h})\end{array}$ & $\begin{array}{l}\text { Längerfristig } \\
\text { abgesagt } \\
\text { (>48h) }\end{array}$ & $\begin{array}{l}\text { Total } \\
\text { Absenzen }\end{array}$ & $\begin{array}{l}\text { Anzahl } \\
\text { Sitzungen } \\
\text { total }\end{array}$ \\
\hline \multicolumn{8}{|l|}{ Periode 1} \\
\hline Absolute Werte & 179 & 10185 & 463 & 535 & 439 & 1437 & 11622 \\
\hline $\begin{array}{l}\text { Mittelwert pro Praxis } \\
\text { pro } 2 \text { Wochen }\end{array}$ & & 56,9 & 2,6 & 3,0 & 2,5 & 8,0 & 65,0 \\
\hline Median & & 58,0 & 2,0 & 2,0 & 2,0 & 8,0 & \\
\hline$\%$ & & 87,6 & 4,0 & 4,6 & 3,8 & 12,4 & 100 \\
\hline \multicolumn{8}{|l|}{ Periode 2} \\
\hline Absolute Werte & 64 & 3355 & 147 & 170 & 229 & 546 & 3901 \\
\hline $\begin{array}{l}\text { Mittelwert pro Praxis } \\
\text { pro } 2 \text { Wochen }\end{array}$ & & 52,4 & 2,3 & 2,7 & 3,6 & 8,5 & 61,0 \\
\hline Median & & 51,0 & 2,0 & 2,0 & 2,0 & 8,0 & \\
\hline$\%$ & & 86,0 & 3,8 & 4,4 & 5,8 & 14,0 & 100 \\
\hline \multicolumn{8}{|c|}{ Total Periode $1+$ Periode 2} \\
\hline Absolute Werte & 243 & 13540 & 610 & 705 & 668 & 1983 & 15523 \\
\hline Mittelwert & & 55,8 & 2,5 & 2,9 & 2,8 & 8,2 & 64,0 \\
\hline$\%$ & & 87,3 & 3,9 & 4,6 & 4,2 & 12,8 & 100 \\
\hline
\end{tabular}


Dauer der ausgefallenen Sitzungen.

\begin{tabular}{|c|c|c|c|c|c|}
\hline & $\begin{array}{l}\text { Anzahl } \\
\text { Praxen }\end{array}$ & $\begin{array}{l}\text { Dauer unabgemeldete } \\
\text { Absenz (Min.) }\end{array}$ & $\begin{array}{l}\text { Dauer kurzfristig (<48h) } \\
\text { abgesagt (Min.) }\end{array}$ & $\begin{array}{l}\text { Dauer langfristig (>48h) } \\
\text { abgesagt (Min.) }\end{array}$ & $\begin{array}{l}\text { Gesamtdauer } \\
\text { der Absenzen (Min.) }\end{array}$ \\
\hline \multicolumn{6}{|l|}{ Periode 1} \\
\hline Absolute Werte & 179 & 22660 & 28120 & 24380 & 75160 \\
\hline $\begin{array}{l}\text { Mittelwert pro Praxis } \\
\text { pro } 2 \text { Wochen }\end{array}$ & & 126,6 & 157,1 & 136,2 & 419,9 \\
\hline Median & & 100 & 120 & 90 & \\
\hline \multicolumn{6}{|l|}{ Periode 2} \\
\hline Absolute Werte & 64 & 7605 & 8700 & 12070 & 28375 \\
\hline Mittelwert & & 118,8 & 135,9 & 188,6 & 443,3 \\
\hline Median & & 115 & 120 & 120 & \\
\hline \multicolumn{6}{|c|}{ Total Periode $1+$ Periode 2} \\
\hline Absolute Werte & 243 & 30265 & 36820 & 36450 & 103535 \\
\hline Mittelwert & & 124,5 & 151,5 & 150,0 & 426 \\
\hline
\end{tabular}

\section{Sitzungszahl}

Die Anzahl der durchgeführten Sitzungen beträgt im Durchschnitt in Periode 1: 56,9 (Median 58,0 ), in Periode 2: 52,4 (Median 51,0) und in beiden Perioden zusammen 55,8. Es wurden in beiden Perioden zusammen total 13540 Sitzungen durchgeführt. Damit fanden pro untersuchte Praxis durchschnittlich wöchentlich 27,9 Sitzungen statt.

Die Ergebnisse der Erhebung betreffend Anzahl ausgefallener Sitzungen sind in Tabelle 2 ersichtlich.

Der gesamte Ausfall aus allen drei Kategorien beträgt in Periode 1 mit 1437 ausgefallenen Sitzungen 12,4\%, in Periode 2 mit 546 ausgefallenen Sitzungen 14,0\%. Die kurzfristig abgesagten und unabgemeldet ausgefallenen Sitzungen (Kategorien 1 und 2) ergeben in Periode $1 \mathrm{mit}$ 998 Sitzungen 8,6\% und in Periode 2 mit 317 Sitzungen $8,2 \%$.

Der gesamte Absentismus beträgt im Mittel beider Erhebungsperioden mit 1983 Sitzungen 12,8\%. Die kurzfristig abgesagten und unabgemeldet ausgefallenen Sitzungen machen mit 1315 Sitzungen insgesamt 8,5\% der vereinbarten Sitzungen aus.

\section{Sitzungsdauer}

Die durchschnittlichen Sitzungsdauern der drei verschiedenen Kategorien abgesagter Sitzungen sind in Tabelle 3 ersichtlich. Die Gesamtdauer der ausgefallenen Sitzungen beträgt im Mittel beider Perioden 426 Minuten. Damit ergibt sich bei durchschnittlich 8,2 wöchentlich ausgefallenen Sitzungen (Tab. 2) eine durchschnittliche Sitzungsdauer der ausgefallenen Sitzungen von 52,0 Minuten.

\section{Diskussion}

Es fällt zunächst die Unterschiedlichkeit des Rücklaufs der beiden Erfassungsperioden auf. In Periode 1 ist der Rücklauf mit über zwei Dritteln hoch. Nach dem Plausibilitätstest blieben 52,4\% der Erfassungsbogen, die repräsentative Ergebnisse ermöglichten.

Der geringe Rücklauf von knapp einem Drittel in der Periode 2 ist durch zwei Faktoren erklärbar: erstens bestand eine zeitliche Verzögerung seit dem Versand der Erhebungsunterlagen bis zur Periode 2 von über zwei Monaten. Zweitens war im Juli die ferienbedingte Abwesenheit vieler Studienteilnehmer sehr hoch. Beide Faktoren wurden aber absichtlich in Kauf genommen, um mit der Erfassung dieser unterschiedlichen Perioden die Abbildung wechselnder Arbeitsbedingungen in der Praxispsychiatrie zu gewährleisten (siehe «Material und Methode»).

Die auffallend geringen Unterschiede in den Ergebnissen rechtfertigen, dass die Durchschnittswerte aus beiden Erhebungsperioden zusammen für die Bestimmung des Ausmasses des Absentismusphänomens herangezogen werden können.

Das Total von 243 ausgewerteten gültigen Erfassungsbögen für die Zeitperioden Periode 1 und Periode 2 zusammen ist mit 14,1\% der Gesamtpopulation der praktizierenden Mitglieder beider psychiatrischen Gesellschaften immer noch beachtlich.

Die Ausschlüsse durch die Plausibilisierung sind mehrheitlich auf die Eingrenzung der Sitzungszahl auf 30 bis 90 pro Erfassungsperiode zurückzuführen. Beschäftigungsgrade unter 50\% 
wurden damit aus der Erhebung ausgeschlossen. Dies erfolgte, um die Erhebungssicherheit und Vergleichbarkeit zu gewährleisten.

Das Absentismusphänomen ist mit gesamthaft 12,8\% unabgemeldeten und abgesagten Absenzen eine gravierende Erscheinung in der psychiatrischen Praxis. Diese Besonderheit bestätigt sich durch diese Studie in eindrücklicher Art.

Von diesem Gesamtprozentsatz betragen die frühzeitig, d.h. 48 Stunden oder früher, abgesagten Sitzungen rund ein Drittel. Damit beträgt der enger gefasste Absentismus der kurzfristig abgesagten oder unabgemeldeten Sitzungen immer noch 8,5\%. Diese kurzfristigen Ausfälle können aus dargestellten strukturellen Gründen in der Psychiatrie nur noch zu einem geringen Teil ersetzt und damit produktiv genutzt werden.

Dass der Absentismus in der Praxispsychiatrie ein wesentliches Ausmass hat, wird eindrücklich belegt. Die Resultate dieser Studie müssen bei der Berechnung der Produktivität in der psychiatrisch-psychotherapeutischen Praxis in vollem Umfang Eingang finden. 